\title{
Psittacosis outbreak in Tayside, Scotland, December 2011 to February 2012
}

C C McGuigan (chris.mcguigan@nhs.net) ${ }^{1}$, P G Mclntyre², K Templeton ${ }^{3}$

1. Directorate of Public Health, NHS Tayside, Kings Cross Hospital, Dundee, United Kingdom

2. Medical Microbiology, Ninewells Hospital and Medical School, Dundee, United Kingdom

3. East of Scotland Specialist Virology Centre, Royal Infirmary of Edinburgh, Edinburgh, United Kingdom

Citation style for this article:

McGuigan CC, McIntyre PG, Templeton K. Psittacosis outbreak in Tayside, Scotland, December 2011 to February 2012 . Euro Surveill. $2012 ; 17(22)$ :pii=20186. Available online: http://www.eurosurveillance.org/ViewArticle.aspx?Articleld=20186

Article submitted on 14 May 2012 / published on 31 May 2012

A Tayside outbreak of psittacosis December 2011-February 2012 involved three confirmed and one probable cases. Confirmed cases were indistinguishable by sequencing of polymerase chain reaction (PCR) products. The epidemiological pattern suggested person-to-person spread as illness onset dates were consistent with the incubation period and no single common exposure could explain the infections. In particular the only common exposure for a healthcare worker case is overlap in place and time with the symptomatic index case.

\section{Outbreak description}

During February 2012, Tayside's Health Protection Team was notified of five cases of pneumonia. These illnesses affected four family members and one healthcare worker (HCW) who had tended the index case. Four of these developed severe symptoms, two requiring intensive care unit (ICU) admission. These four had complement fixation tests (CFT) suggesting infection with a Chlamydophila species. Although speciation was not possible at this stage, the time interval of one to 22 days between the symptom onset of consecutive cases, suggested person-to-person spread. An outbreak of Chlamydophila pneumoniae infection therefore seemed likely. Pending identification, the outbreak response proceeded on this basis. By mid-February C. psittaci was confirmed by polymerase chain reaction (PCR).

\section{Background}

Psittacosis is a systemic infectious disease caused by Chlamydophila psittaci. Usual features include fever, malaise, unproductive cough, headache and atypical pneumonia. The incubation period is one to four weeks [1]. Since its first description in 1879 [2], epidemics occurred during the next century. Where identified, the source of such outbreaks and infections was zoonotic, and predominantly avian but not necessarily psittacine. For example, large outbreaks occurred among poultry workers [3]. Subsequently, these have become rare, as avicultural hygiene has intensified. In Scotland, up to 10 sporadic cases per year were notified (no outbreaks) in the past 10 years (Table) [4]. We have found no case described in the literature where person-to-person spread has accounted for cases of psittacosis, although person-to-person transmission has evidently been suggested but not proven [5].

\section{Outbreak investigation and results}

During a series of outbreak management team (OMT) meetings, results were assessed and further investigation directed. Awareness raising among Tayside medical practitioners aimed to increase case ascertainment. The investigation progressed on three fronts: epidemiological, microbiological and environmental.

\section{TABLE}

Total number of cases of Chlamydophila psittaci infections notified annually, Scotland, 2001-2011 (n=27)

\begin{tabular}{|c|c|c|c|c|c|c|c|c|c|c|c|}
\hline Year & 2001 & 2002 & 2003 & 2004 & 2005 & 2006 & 2007 & 2008 & 2009 & 2010 & 2011 \\
\hline Number of cases & 2 & 10 & 1 & 4 & 0 & 0 & 1 & 1 & 2 & 5 & 1 \\
\hline
\end{tabular}

Source: Health Protection Scotland (HPS) (Lynda Browning, personal communication, 23 May 2012) [4]. 
Time of symptom onset and clinical course of probable and confirmed cases, psittacosis outbreak in Tayside, Scotland, December 2011-February $2012(n=4)$

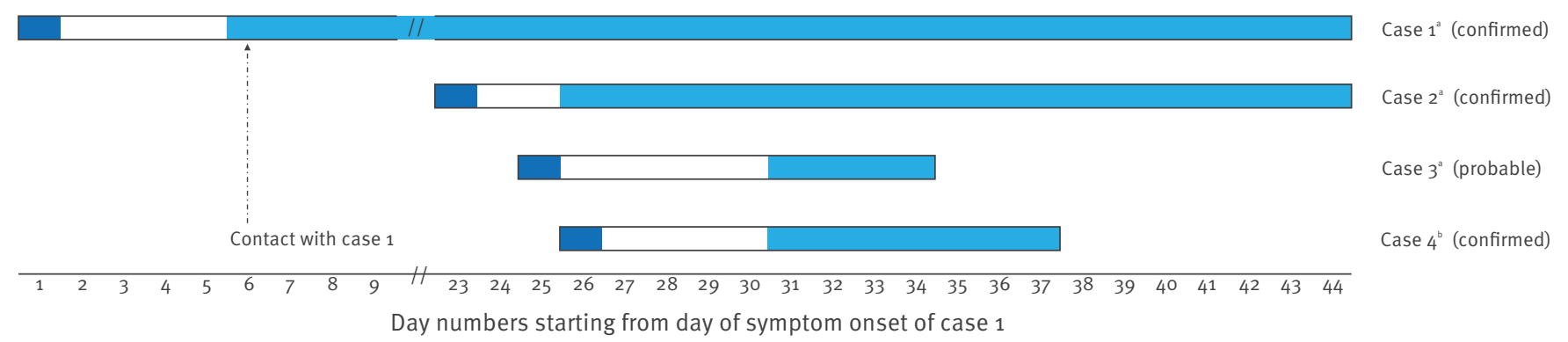

${ }^{b}$ Case 4 , a healthcare worker, had contact with case 1 on the sixth day of case 1 's illness, as indicated by an arrow.

Epidemiological investigation

A modified Centers for Disease Control and Prevention (CDC) case definition [6] was agreed. To be considered, cases must have compatible clinical illness. All notified cases were interviewed about their illness, contacts and relevant possible exposures. Confirmed cases had either Chlamydophila species detected in respiratory secretions (by culture or PCR) or a fourfold or greater increase in antibody (IgG or IgM) to Chlamydophila species (to a reciprocal titre of 32 between paired acute- and convalescent-phase serum specimens taken at least two weeks apart) by CFT. Cases which were epidemiologically linked to a confirmed case were considered probable, given an antibody (IgG or IgM) titre of 256 or greater, and possible given one of 32 to 128 (all by CFT in a serum specimen taken after symptom onset).

Applying this, by 22 February 2012, the outbreak involved three confirmed, one probable and two possible cases, with the index case having had onset of illness in late December 2011. The figure describes the time of onset and clinical course for confirmed and probable cases. These comprised three female and one male with an age range of 41 to 65 years.

A further two possible cases were identified: a family member with mild respiratory illness and an unrelated patient from the same ICU as the index case.

\section{Microbiological investigation}

Initial investigations used CFT performed according to standard methods using antigen obtained from Launch Diagnostics, Longfield, Kent, United Kingdom (UK) [7]. The CFT antigen is a chlamydia group specific antigen. The test detects total complement fixing antibody: both IgG and IgM.
Real-time PCR was performed using in house assay on respiratory samples which were initially used for investigations for respiratory viruses. The screen for Chlamydophila species was an assay targeted to $16 \mathrm{~S}$ ribosomal sequences. Any positive sample was further investigated by specific real-time PCR to C. psittaci or C. pneumoniae targeting a different region of the $16 \mathrm{~S}$ ribosomal sequence. This enabled determination of which Chlamydophila species was involved in a case.

Of the confirmed cases, two showed a rising CFT titre, one a static raised titre. All were PCR positive. Sequence analysis of the outer membrane protein $A$ (ompA) gene showed $100 \%$ similarity between these C. psittaci strains. The probable case had a static CFT titre above 256 and was PCR negative. Possible cases had static titres of 64 to128 and were PCR negative.

\section{Environmental investigation}

Extensive cartographical and field searches were made for possible avian sources of infection. These were directed by information gleaned from interviews with cases. Workplaces and residences of cases were plotted on an Ordnance Survey map. Cases 2 and 3 lived together a kilometre from case 1 . Case 4 resided a further ten kilometres west. Although not within any of the cases' respective place of residence, two pigeon coops and a cage of small birds were found in the neighbourhood of where cases 1, 2 and 3 lived. None were within $500 \mathrm{~m}$ of case 1 , but as these could be considered a plausible source, faecal samples were taken for PCR analysis.

The index case's pet dog was reported to have rolled in the remains of a dead bird in December. Also, this 
case's workplace was reported to be affected by a large number of gulls. Searches in both areas revealed insufficient sample material. On veterinary recommendation (included in the OMT), a PCR analysis of a pooled canine faecal sample was done, using an unpublished method, developed at the UK Animal Health and Veterinary Laboratories Agency, Weybridge. This PCR detects the presence of $C$. psittaci and $C$. abortus and was negative.

No environmental source of any Chlamydophila species was revealed by environmental investigations. This is not unusual [8].

\section{Control measures}

Since the source of the infection was thought to be a pathogen which was not readily transmissible from person-to-person, standard infection control measures were recommended for those HCWs and other people in contact with cases.

\section{Discussion and conclusion}

The main issue in this outbreak is the picture of person-to-person spread. The authors can find no description of this in psittacosis. Incubation ranging from one to four weeks implies up to 21 days between shortest and longest. The longest gap between onset of confirmed cases was 25 days. While the cases amongst the extended family might be explained by a putative persistent source to which family members were sequentially exposed (e.g. a geographical, not temporal, point source), case 4 (the HCW) cannot.

Since cases 1 to 3 were members of an extended family and had extensive and frequent contact with each other (especially over the winter holiday season) it was not possible to retrospectively identify particularly significant 'mutual exposure events'. However, shared exposures between case 4 and the others were sought. The only spatial-temporal overlap was with case 1 and occurred during the admission of case 1 to the ward where case 4 worked. Case 4's duties included personal care (not invasive procedures). Conceivably, case 4 may have been exposed while caring for case 1 who required intensive medical support and investigation. Since it was not possible to explore direct contact between the two cases, it is uncertain what such exposure might be.

It is difficult to explain all cases in this outbreak by exposure to a common non-human source. While inconclusive, features consistent with person-person spread are demonstrated. In our view, clinicians and public health specialists should therefore keep an open mind to the possibility of person to person spread of psittacosis despite the received opinion that this generally does not occur.
References

1. Heymann DL, editor. Control of communicable diseases manual. 19th ed. Washington DC: American Public Health Association; 2008.

2. Vanrompay D, Ducatelle R, Haesebrouck F. Chlamydia psittaci infections: a review with emphasis on avian chlamydiosis. Vet Microbiol. 1995;45(2-3):93-119.

3. Gaede W, Reckling KF, Dresenkamp B, Kenklies S, Schubert E, Noack U, et al. Chlamydophila psittaci infections in humans during an outbreak of psittacosis from poultry in Germany. Zoonoses Public Health. 2008;55(4):184-8.

4. Health Protection Scotland (HPS). Chlamydia psittaci, Scotland, Annual Total as at 28 June 2011. Glasgow: HPS. [Accessed 24 May 2012]. Available from: http://www. documents.hps.scot.nhs.uk/giz/10-year-tables/psittaci.pdf

5. Centers for Disease Control and Prevention. Compendium of measures to control Chlamydia psittaci infection amons humans (psittacosis) and pet birds (avian chlamydiosis), 2000 and Compendium of animal rabies prevention and control, 2000. National Association of State Public Health Veterinarians, Inc. MMWR Recomm Rep. 2000;49(RR-8):3-17.

6. Centers for Disease Control and Prevention. Compendium of measures to control Chlamydia psittaci infection among humans (psittacosis) and pet birds (avian chlamydiosis), 2000 and Compendium of animal rabies prevention and control, 2000. National Association of State Public Health Veterinarians, Inc. MMWR Recomm Rep. 2000;49(No. RR-8):5

7. Grist NR, Ross CA, Bell EJ. Diagnostic Methods in Clinical Virology. Second edition. Blackwell Scientific Publications: Oxford; 1974 .

8. Mulder MM, Heddema ER, Pannekoek Y, Faridpooya K, Oud ME, Schilder-Tol E, et al. No evidence for an association of ocular adnexal lymphoma with Chlamydia psittaci in a cohort of patients from the Netherlands. Leuk Res. 2006;30(10):1305-7. 